\section{Changes in the urban landscape at the end of the 20th century: An overview of the special issue}

\author{
Rita Schneider-Sliwa, Basel
}

Postindustrial, postmodern society and a globalizing economy create spaces of extraordinary multiplicity. New real, perceived and lived spaces emerge in metropolitan and inner city areas as manifestations of societal change and economic restructuring. This issue focuses on the transformation of the contemporary urban landscape. The articles address new types of urban and social space and the planning of urban areas.

A. Kampschulte's and R. Strassmann's paper on the restructuring of the urban and suburban economy is based upon the notion of a dialectic between the global and the local. It traces effects in a metropolitan area economy as a result of the powerful forces of globalization. Based upon the theoretical literature on the local area impact of global economic restructuring, the authors check the ground reality, using the Basle metropolitan area as an example. Functional and social segregation processes (A. Odermatt) are also factors leading to the remapping of the urban economic, social and cultural landscape. Using the Zurich metropolitan area as a case study, the fiscal drain on central cities is discussed that results from the preference for suburban living. Des Edge-Cities en Suisse? (P. DESSEMONTET) is concerned with the effects of suburbanization on the urban periphery. The rise of new suburban employment centers, although on a much smaller scale as compared to Edge-Cities in the US, gives rise to new concerns and research in small countries like Switzerland: suburban commercial developments may threaten classical inner city economies long before they may reach "American dimensions". As suburban landscapes are recomposed by new commercial developments, so are inner city areas. Restructuring by exclusion processes, society and polity is the theme of A. DE CUNHA's paper on urban poverty in Switzerland. Exclusion by individual adjustment mechanisms as well as public policy are addressed. Public policy as manifest in urban planning measures is the concern of J. RuEGG's article that raises the question whether or not urban planning measures are helpful to urban development. Using the case of a development project in Toronto he argues that in powerful conflicts of interest regarding the use of urban land existing land use regulations may not hold up to pressure. How the ground can be prepared for inner city redevelopment is discussed by $\mathrm{B}$. Belina. He turns attention to the subtle mechanisms at work: the "criminalizing" of urban space. Ideologically motivated, this making of urban images serves to eliminate unwanted elements from areas of potential.

\section{Die Transformation von Stadt- landschaften im ausgehenden 20. Jahrhundert: Das Themenheft im Überblick}

Die postindustrielle, postmoderne Gesellschaft und die Globalisierung haben Räume von ausserordentlicher Vielfalt hervorgebracht. Neue reale sowie wahrgenommene und erlebte Räume entstehen in Agglomerationen und innerstädtischen Gebieten als Manifestation dieser gesellschaftlichen Veränderungen und wirtschaftlichen Umstrukturierungen. Dieses Themenheft ist der Transformation der modernen Stadtlandschaft gewidmet. Behandelt werden neue Formen urbanen und städtischen sozialen Raumes sowie die Planung für solche Räume.

A. Kampschultes und R. Strassmanns Aufsatz über die Restrukturierung der Stadt-UmlandÖkonomie geht von einer Dialektik zwischen Globalem und Lokalem aus und geht den lokalen Auswirkungen der Globalisierung nach. Aufgrund der theoretischen Postulate zu räumlichen Auswirkungen der Globalisierung wird ein «ground check» der empirischen Realität am Beispiel Basels vorgenommen. Die funktionale und soziale Entmischung in einem Agglomerationsraum (A. OdermatT) ist ein weiterer Faktor in der sozialen, wirtschaftlichen und kulturellen Umgestaltung der Stadtlandschaft. Am Beispiel des Grossraums Zürich wird der Steuerabfluss der Kernstadt als Begleiterscheinung der Präferenz für suburbanes Wohnen behandelt. «Des Edge-Cities en Suisse?» (P. Dessemontet) widmet sich den Effekten der Suburbanisierung auf die urbane Peripherie. Das Aufkommen solcher suburbaner Arbeitsplatzzentren gibt Anlass zu neuen Problemstellungen: Auch wenn diese Zentren viel kleinere Dimensionen haben als amerikanische Edge-Cities, werden ihre Auswirkungen auf die Ökonomie der Kernstädte in einem kleinen Land wie der Schweiz auch viel früher fühlbar. So wie der suburbane Raum durch Entwicklungen «auf der grünen Wiese» ein neues Gesicht erhält, wird auch der Innenstadtraum neu geprägt. Die Restrukturierung durch Ausgrenzungsprozesse, die Gesellschaft und 
die planungspolitische Ebene sind das Thema des Beitrages zur städtischen Armut in der Schweiz (A. DE CunHa). Er umreisst sowohl den freiwilligen Rückzug betroffener Individuen als auch die Ausgrenzung durch die öffentliche Verwaltung. Deren Politik, die sich zum Beispiel in Stadtplanungsmassnahmen manifestiert, ist Anliegen der Arbeit von J. Ruegg. Er stellt die Überlegung an, ob Stadtplanungsreglemente in innerstädtischen Gebieten, die unterschiedlichen Interessensansprüchen ausgesetzt sind, wirklich hilfreich sind. Am Beispiel eines Erschliessungsprojektes in Toronto wird argumentiert, dass bestehende Flächennutzungsbestimmungen in solchen Gebieten nicht immer standhalten können. Auf welche Weise der Weg für Innenstadtaufwertung geebnet werden kann, diskutiert B. BELINA. Er weist auf die subtilen Mechanismen hin, die hier am Werk sind: die «Kriminalisierung» von Stadträumen. Nach dieser ideologisch motivierten Gleichsetzung gewisser urbaner Räume mit Kriminalität, die erst ein bestimmtes Image von einem Stadtteil schafft, können Gebiete mit Profitpotential von unerwünschten Personen freigehalten werden.

\section{La transformation des paysages urbains vers la fin du vingtième siècle: Résumé de la revue thématique}

La société postindustrielle et postmoderne, ainsi qu'une économie mondialisée génèrent des espaces aux facettes multiples. De nouveaux espaces réels, perçus et vécus émergent dans des agglomérations et des centre-villes en tant que manifestations de changements de la société et de restructuration économique. Ce volume se penche sur la transformation du paysage urbain contemporain. Les articles parlent de nouveaux espaces urbains et sociaux, ainsi que l'aménagement de zones urbaines.

La contribution de A. Kampschulte et R. StrassMANN sur la restructuration de l'économie urbaine et de la périphérie se base sur des notions de dialectique entre l'espace global et l'espace local. Elle retrace les effets des forces de la globalisation sur l'économie d'une agglomération. Basée sur la littérature théorique concernant l'impact local de la restructuration économique globale, les auteurs se réfèrent à la réalité en utilisant l'agglomération de Bâle comme exemple. La ségrégation fonctionnelle et sociale (A. ODERMATT) représente un facteur supplémentaire qui redessine le paysage urbain, social, et économique. P. DessemonTET dans son article des «Edge-Cities» en Suisse. L'augmentation de nouveaux centres d'emploi en périphérie des villes, bien que moins accentuée qu'aux EtatsUnis, provoque de nouvelles préoccupations et de nouveaux axes de recherche dans de petits pays comme la Suisse. Le développement commercial à la périphérie des villes pourrait menacer le tissus économique classique des centre-villes bien avant d'atteindre des proportions «à l'américaine». Alors que le paysage de la périphérie se transforme par de nouveaux développements commerciaux, celui des centre-villes se transforme également. Les processus d'exclusion sociale par les restructurations économiques fait l'objet de l'article de A. DE CUNHA sur la pauvreté urbaine en Suisse. L'exclusion par des mécanismes individuels d'ajustement ainsi que la politique des autorités communales y sont développées. La politique de l'aménagement du territoire décrite par J. RUEGG aborde la question de savoir si les mesures d'aménagement urbain sont en fin de compte utiles au développement urbain. Le cas d'étude d'un projet de développement à Toronto met en évidence le fait que des conflits d'intérêts majeurs concernant l'utilisation de terrains urbains peuvent rendre à néant les réglementations de l'aménagement du territoire qui sont en vigueur. B. Belina évoque la manière dont on pourrait mieux préparer le terrain pour un redéveloppement des centre-villes. Il se penche sur des mécanismes incidieux qui se dessinent dans certains grands centres urbains, notamment la «criminalisation» de l'espace urbain. Cette image idéologique sert à se débarasser d'éléments indésirables dans des zones où il existe un potentiel de développement économique.

Prof. Dr. phil. Rita Schneider-Sliwa, Abteilung Humangeographie/Stadt- und Regionalforschung, Departement Geographie, Universität Basel, Klingelbergstrasse 16, CH-4056 Basel. 